\title{
Consecutive regression of MALT lymphomas coexisting in the pharyngeal and gastric tissue after the eradication of Helicobacter pylori
}

Giuseppe Ivan Potente, Salvatore Nisticò'

ASP Catanzaro, Soveria Mannelli Hospital, Department of Medicine Laboratory, Clinical Microbiology Unit I ASP Catanzaro, Lamezia Terme Hospital, Department of Medicine Laboratory, Clinical Microbiology Unit

Key words: MALT lymphoma, Helicobacter pylori, pharyngeal tissue

\section{SUMMARY}

The stomach is one of the most common organs in which mucosa-associated lymphoid tissue (MALT) lymphoma develops. It is well established that Helicobacter pylori $(\mathrm{Hp})$ infection plays a major role in the development of gastric MALT lymphoma and that the presence of $\mathrm{Hp}$ in the gastric mucosa is connected with mucosa-associated lymphatic tissue (MALT).The same tissue is located in the oral cavity and pharynx in Waldayer's circuit. Recently, the oral cavity was proposed as an extragastric reservoir of $\mathrm{Hp}$ infection. We report the case of a 79-year-old female patient with concomitant pharyngeal (MALT) lymphoma and Hp-related gastric MALT lymphoma. Gastric MALT lymphoma was detected both through endoscopic examination as well as in biopsies.

Pharyngeal MALT lymphoma was also detected in biopsies. Hp has been recognized in the gastric mucosa by positive serum $H$. pylori antibody and urease tests. Treatment of the $\mathrm{Hp}$ infection in our patient using antibiotics led to the regression of both lesions. This is the first case report on the regression of a pharyngeal MALT lymphoma after Hp eradication.

\section{INTRODUCTION}

The stomach is one of the most common organs in which mucosa-associated lymphoid tissue (MALT) lymphoma develops. It is well established that Helicobacter pylori (Hp) infection plays a major role in the development of gastric MALT lymphoma and that the presence of $\mathrm{Hp}$ in the gastric mucosa is connected with mucosa associated lymphatic tissue MALT.

The same tissue is located in the oral cavity and pharynx in Waldayer's circuit. Recently, the oral cavity was proposed as an extragastric reservoir of Hp infection. We report the case of a 79-yearold female patient with concomitant pharyngeal MALT lymphoma and Hp-related gastric MALT lymphoma. Gastric MALT lymphoma was detected both through endoscopic examination as well as in biopsies. Pharyngeal MALT lymphoma was also detected in biopsies.

$\mathrm{Hp}$ has been recognized in the gastric mucosa by positive serum $\mathrm{Hp}$ antibody and urease tests. Treatment of the Hp infection in our patient using antibiotics led to the regression of both lesions. This is the first case report on the regression of a pharyngeal MALT lymphoma after Hp eradication.

Helicobacter pylori ( $\mathrm{Hp})$ is a spiral, microaerophilic, Gram-negative bacterium. It is considered to be the most common chronic bacte- rial infection in humans (4). The prevalence estimates to range from 40 to $80 \%$. Hp plays a major role in the pathogenesis of chronic gastritis, duodenal and gastric ulcers, adenocarcinoma and gastric lymphoma (6).

The most common primary lymphoma of the gastrointestinal tract is B-cell lymphoma arising from mucosa-associated lymphoid tissue, known as MALT lymphoma. Although the stomach is the most common site of MALT lymphoma, other sites have been described and numerous case reports on MALT lymphoma simultaneously involving 2 or more organs have been reported. There are no cases reported of patients with concomitant pharyngeal (MALT) lymphoma and Hprelated gastric MALT lymphoma.

Recently, Hp is supposed to be present in the oral cavity. Hp has been detected in both dental plaque and saliva (1).

For this reason, great interest has recently been focused on the detection of Hp presence in tonsillar and adenoid tissue.

The immunological changes caused by $\mathrm{Hp}$ in the stomach mucosa have recently been explained (3). More detailed data about Hp's effect on the oropharyngeal mucosa is not available. In this report, we record the results of our study of a patient with an oropharyngeal neoformation and dyspeptic syndrome.

\section{Corresponding author: Giuseppe Ivan Potente}

Department of Medicine Laboratory, Clinical Microbiology Unit, Soveria Mannelli Hospital

Phone: +393207680 I 26

E-mail: ivanpotente@libero.it 


\section{CASE REPORT}

A 79-year-old Italian woman came to our clinic in 2008, complaining of abdominal discomfort and distension. She had no significant medical history. A t.c. examination revealed a protruding neoformation in the oropharyngeal cavity measuring 2.6 $x 1.7 \mathrm{~cm}$, and a biopsy specimen of the neoformation showed MALT lymphoma. This diagnosis was made at the Santa Chiara Hospital in Pisa; then a cycle of radiotherapy was suggested. During our observation, the patient had already completed the first cycle of radiotherapy and was waiting to start a second one. We performed the anti-Hp IgG antibodies and anticitoxine IgG antibodies, to evaluate whether the dyspeptic suspicion could be associated to Hp infection.

After getting positive results from both tests confirming the ongoing infection, a stool specimen was tested for Hp antigens (met E.I.A). Even though this latter test was positive, an EGDS was carried out in order to have a complete diagnosis. The result highlighted the presence of an active chronic-erosive gastritis with glandular atrophy and a follicular hyperplasia of the malt correlated to the infection of the Hp. This was evidenced by the histological report of the Lamezia Terme hospital, Department of Anatomical Pathology. Once the diagnostic process was completed and the correlation between the presence of the gastric lymphoma and the pharyngeal and after observation of the patient that meanwhile had undergone radiotherapy, we suggested the eradication therapy for $\mathrm{Hp}$ following the described therapeutic scheme: association of ciprofloxacin 500 mg+amoxicillin 1gr, morning and evening for 14 days, omeprazole $20 \mathrm{mg}, 1$ tablet in the morning for 60 days, lactobacilli, morning and evening for 20 days. After 60 days from the last antibiotic therapy, we carried out a check test looking for the $\mathrm{Hp}$ antigen in a stool specimen to see if the eradication therapy was working, but the test was negative. Also the test on the oropharyngeal neoformation showed the regression of the tumor.

The EGDS done 12 months after interruption of the therapy showed the regression of the inflamed gastric mucosa with the disappearance of the MALT lymphoma. The clinical condition of the patient had improved, and the patient had no dyspeptic disorder. The search for Hp antibodies in stool samples was carried out several times over the years to verify the absence of recurrences and it has always shown a negative result.

Today, the clinical condition is good and the latest check showed a good general condition, without signs of recurrence of the lymphatic pathology over the last 4 years until present. The 4-year follow-up gives us the possibility to confirm that the anti $\mathrm{Hp}$ eradication therapy could heal not only the MALT gastric lymphoma, but also the oropharyngeal one. Moreover, we confirm that the $\mathrm{Hp}$ is a microorganism capable of generating and sustaining neoplastic processes in established gastric tissue as well as elsewhere.

So we are quite sure about the scientific assumption that patients affected by the malt extragastric lymphoma should always suspect the presence of a correlated $\mathrm{Hp}$ gastritis. For this reason, they always have to be checked.

\section{DISCUSSION}

There is a scientifically proven correlation between $\mathrm{Hp}$ infection and the onset of MALT lymphoma type. Data in literature shows that in patients with gastric MALT lymphoma there is a contextual evidence of $\mathrm{Hp}$ infection in the active phase and that the eradication of $\mathrm{Hp}$ matches a regression of MALT lymphoma in up to $92 \%$ of cases. The correlation is clear from a pathological point of view according to the specific activities carried out by MALT, i.e. the processing of the antigen that comes from the outside into the cells of the gastric mucosa and plays a role in the immune defense of the gastro-intestinal lymphatic system.

Chronic infection with $\mathrm{Hp}$ is the only cause currently known to induce gastric MALT lymphoma (3). An atypical localization of the MALT lymphoma is evident in our study. Besides stomach problems the patient had also a throat lesion not yet described in literature.

The anamnesis revealed the existence of such gastric dyspeptic symptoms that led us to treatment instantly in order to confirm the diagnostic assumption with histological evidence of gastric MALT lymphoma associated with chronic erosive gastritis with $\mathrm{Hp}$ infection.

The patient underwent therapy for Hp eradication using a combination of antibiotics (ciprofloxacin and amoxicillin); this therapy differs from the classic approach based on scientific evidence and favoring a combination of two bactericidal drugs, rather than a bactericidal and bacteriostatic one, in particular when the Hp infection evolves into a chronic infection.

Of course the use of omeprazole as a guideline has completed the effect, allowing the patient to complete regression of both lesions and achieve a better quality of life. The patient has been followed through regular examination for a long period of time in order to give the highest scientific validation. The study confirms the strong correlation between the occurrence of MALT-type lesions and infection from Hp. Moreover, it shows the possibility of an involvement of new anatomical 
sites. Based on these findings, we confirm that in most cases the eradication of Hp leads to a regression of MALT-type lesions; this statement is supported by strong evidence we have gathered after more than over a period of three year follow-up.

\section{REFERENCES}

1. Kimiyuki H, Takashi N, Tsutomu C, Yumi M, Takane K. Regression of MALT lymphomas coexisting in the duodenal bulb and stomach by eradication of Helicobacter pylori. Journal of Gastroenterology. 2002; 37: 288-92.

2. Lukes P, Astl J, Pavlik E, Potuznikova B, Sterzl I, Betka J. Helicobacter pylori in tonsillar and adenoid tissue and its possible role in oropharyngeal carcinogenesis. Folia Biologica. 2008; 54: 33-9.
3. Masahiro I, Kazuichi O, Kazushige U, et al. Characteristic of gastric B-cell lynphoma of mucosaassociated lymphoid tissue involving multiple organs. Journal of Gastroenterology. 2004; 39: 739-46.

4. Seiichi K, Kyoko O, Hitoshi S, et al. Helicobacter heilmannii infection in a child after successful eradication of Helicobacter pylori: case report and review of literature. Journal of Gastroenterology. 2005; 40: 94-7.

5. Tsutomu C, Hiroshi S, Hiroyuki M, Yoshio W, Kazuichi O. Host factors are important in determining clinical outcomes of Helicobacter pylori infection. Journal of Gastroenterology. 2006; 41: 1-9.

6. Yosuke K, Toshiyuki M, Takashi H, et al. Deep infiltrative low-grade MALT colonic lymphomas that regressed as a result of antibiotic administration: endoscopic ultrasound evaluation. Journal of Gastroenterology. 2005; 40: 843-7. 